\title{
Paysage-lumière et environnement urbain nocturne
}

La version définitive de l'article a été publiée dans Espaces et Sociétés, n¹45, 2011/3.

\author{
Sandra Mallet \\ EA2076 Habiter-IATEUR \\ URCA \\ sandra.mallet@univ-reims.fr
}

\begin{abstract}
Résumé
Les nuits urbaines françaises se transforment fortement depuis une trentaine d'années. En parallèle, les discours autour de l'urbanisme-lumière font consensus auprès des acteurs de l'éclairage public et semblent acheminer les villes vers des éclairages plus qualitatifs et conçus à une échelle plus large. Dans ce cadre, nous interrogeons les manières dont est traitée la pollution lumineuse au sein des espaces urbains. II ressort de nos analyses menées à Paris, Saint-Denis et Bordeaux des confusions régnant autour de cette expression et des moyens réduits et quasi-exclusivement technicistes pour parvenir à la diminuer. L'hypothèse est que cela est lié à des réflexions se positionnant en termes de paysage et non d'environnement nocturne, délaissant alors certains aspects du fonctionnement des espaces urbains nocturnes. Des exemples pris autour des usages spatiaux et pouvant participer à une meilleure maîtrise de cette pollution sont alors mis en évidence.
\end{abstract}

Mots-clés : pollution lumineuse, espaces urbains, paysage nocturne, urbanisme-lumière, éclairage public 
Les nuits urbaines françaises se sont progressivement animées sous l'effet des éclairages, de la division du temps en heures, du travail à horaires décalés et des loisirs. Or depuis une trentaine d'années, les mutations s'accélèrent [Gwiazdzinski, 2002]. De plus en plus investie par les activités humaines, envahie par les éclairages et convoitée par divers acteurs, la nuit fait dorénavant l'objet de débats multiples et se situe au cœur d'enjeux économiques, politiques, sociaux et environnementaux. Les limites entre le jour et la nuit paraissent de plus en plus floues et il semble se créer une continuité inédite entre ces deux périodes. De ces constats naissent des débats sur son aménagement. La nuit représente pour certains la "dernière frontière " et un espace à conquérir tandis que pour d'autres, elle est désormais " espace à préserver ».

L'éclairage des villes étant l'une des conditions majeures de la vie urbaine nocturne, changeant nos rapports au temps et transformant radicalement le cadre physique de la nuit, il est intéressant de s'interroger sur ses rapports à la ville nocturne dans le cadre de ces mutations. D'autant plus que, à partir de la fin des années 1980, les concepteurslumière puis les éclairagistes ${ }^{1}$, s'accordent à dire qu'un "tournant " dans la façon d'éclairer les villes s'est produit [Narboni, 1997]. L'« urbanisme-lumière " serait ainsi né, correspondant à une nouvelle conception de l'éclairage urbain. Celui-ci se veut plus qualitatif, jouant sur les images de la ville et ses ambiances et conçu à une échelle plus large, s'attachant à l'ensemble d'une ville ou d'un quartier. II se base sur des documents visant à coordonner les futures opérations d'éclairage et éviter les actions au coup par coup (le plan-lumière, le Schéma Directeur d'Aménagement Lumière (Sdal) et la chartelumière). L'éclairage s'affirme alors comme un véritable outil d'urbanisme et d'aménagement, pouvant pleinement participer à l'organisation des espaces urbains. La plupart des villes françaises, même de taille modeste, en sont aujourd'hui dotées.

Les différents acteurs de l'éclairage public estiment avoir développé des savoir-faire améliorant la qualité des espaces urbains nocturnes. Nous interrogeons ici le traitement actuel par les collectivités territoriales de la " pollution lumineuse ", expression suscitant de nombreux débats depuis les années 1980. De quelles manières les politiques d'éclairage façonnent-elles le paysage nocturne ? De quelles façons comprennent-elles la pollution lumineuse et en quoi tentent-elles alors de la réduire ?

Nous souhaitons mettre en avant le paradoxe existant entre, d'un côté, des discours généraux prônant des éclairages de plus en plus " qualitatifs » et, de l'autre, le traitement de la pollution lumineuse qui, lui, est essentiellement techniciste. Ce paradoxe naît, selon nous, d'approches en matière d'urbanisme-lumière s'intéressant principalement au paysage reconstruit par la lumière, entendu comme une toile de fond, un décor conçu pour être regardé et qui se donne à voir de l'extérieur. Bien d'autres aspects de l'environnement nocturne s'en trouvent alors délaissés. Cette analyse ressort de l'étude de trois villes aux dynamiques pourtant bien différentes:

- Paris, "Ville lumière " dont l'imaginaire est fortement associé à la nuit ;

- Saint-Denis, commune de banlieue parisienne confrontée à des problèmes d'insécurité et des populations qui investissent peu l'espace nocturne [Bellavoine, 2005];

- Bordeaux, dont les nuits ont radicalement changé de visage avec l'arrivée d'un nouveau maire en 1996 qui a instauré une ambitieuse politique d'éclairage [Mallet, 2010].

\footnotetext{
${ }^{1}$ Le concepteur-lumière est à distinguer de l'éclairagiste. Les compétences de l'éclairagiste relèvent surtout du savoir-faire technique tandis que les spécificités du concepteur résident dans l'invention, la création [Fiori, Regnault, 2006]
} 
II repose sur deux types de sources principales. D'une part, des sources écrites, majoritairement composées de documents produits ou commandés par les services en charge des éclairages publics de ces Villes, d'archives des conseils municipaux ainsi que d'articles de presses nationales, locales et spécialisées. D'autre part, des entretiens semidirectifs (17 au total) avec les principaux acteurs: éclairagistes et élus en charge de l'éclairage public au sein de ces collectivités, concepteurs-lumière indépendants et associations d'éclairagistes et de concepteurs-lumière.

Nous étudierons les manières dont la pollution lumineuse est définie et traitée par les politiques-lumière du début des années 1990 à 2009, avant de mettre en évidence les lacunes de ces politiques en matière de pollution lumineuse.

\section{La pollution lumineuse au carrefour des confusions}

\section{Les effets néfastes des éclairages artificiels}

Le terme de pollution lumineuse est loin de faire consensus au sein de l'ensemble des acteurs de l'éclairage public et provoque de nombreux débats. Rappelons que la reconnaissance de la pollution lumineuse, qui émerge depuis les années 1970, naît d'un mécontentement de la part des astronomes face à l'intensification globale des éclairages urbains. En effet, les éclairages créent un voile lumineux au-dessus de chaque ville et interdisent aux astronomes de percevoir les étoiles depuis un poste d'observation trop proche d'une grande ville. A titre d'exemple, un halo de lumière couvre le ciel jusqu'à 150 kilomètres de la Tour Eiffel et jusqu'à une cinquantaine de kilomètres du centre de Bordeaux. Les astronomes forment alors des associations regroupant professionnels et amateurs pour protester contre ce "trop-plein » d'éclairage : I'association Dark Sky est ainsi créée aux Etats-Unis en 1998 et l'ANPCEN² est fondée en 1999 en France -soit dans une période de forte diffusion de l'urbanisme-lumière.

L'existence de la pollution lumineuse est désormais reconnue par la communauté scientifique. Ainsi, les écologues tentent de plus en plus de mesurer l'impact de l'éclairage artificiel sur la faune, la flore et l'Homme. Les américains Travis Longcore et Catherine Rich distinguent ainsi deux types de pollution lumineuse [Longcore, Rich, 2004]. La première est dite "astronomique" : elle se rapporte à la vue que nous avons du ciel nocturne, à la disparition des étoiles. La seconde est dite "écologique" : que la diffusion de la lumière se perde vers le ciel ou soit relativement bien dirigée vers le sol, elle altère irrémédiablement les écosystèmes.

La " pollution lumineuse écologique " concerne des espèces de toutes tailles et peut avoir des effets directs et indirects, dont beaucoup sont encore méconnus. Les écologues repèrent, au niveau des écosystèmes, que les éclairages perturbent les comportements de nombreux animaux, en créant notamment des effets d'orientation et de populations : ils modifient les déplacements, les migrations et peuvent attirer ou faire fuir animaux et insectes. La lumière artificielle bouleverse la reproduction et la communication à l'intérieur d'une même espèce. Elle perturbe aussi les interactions entre espèces, en particulier les rapports entre prédateurs et proies. Elle favorise, en outre, certains

\footnotetext{
${ }^{2}$ Association Nationale pour la Protection du Ciel et de l'Environnement Nocturnes
} 
animaux et insectes au détriment d'autres, ce qui change les donnes de la compétition qui peut les opposer. L'ensemble a alors des conséquences considérables sur les écosystèmes. En ce qui concerne l'être humain, les chronobiologistes ont montré que les éclairages peuvent provoquer des dysfonctionnements internes. Ils perturbent le rythme nycthéméral de l'homme, né de l'alternance naturelle entre le jour et la nuit : la sécrétion de mélatonine est d'autant plus forte que la lumière est faible. Or cette hormone est fondamentale à presque tous les organismes animaux. Sa sécrétion avance ou retarde l'endormissement. Son action ne s'arrête pas là : elle aurait aussi des effets sur la reproduction, la fabrication des spermatozoïdes chez l'homme ainsi que sur le cycle menstruel de la femme. Elle détiendrait également des propriétés anticancéreuses : la suppression de cette hormone par la lumière artificielle est probablement la cause d'une augmentation très importante du taux de cancer du sein chez les femmes travaillant la nuit [Kwiatkowki et al., 2004].

La pollution lumineuse atteint l'être humain sur différents plans : celui de sa santé, mais aussi au niveau culturel et philosophique. Hubert Reeves, astrophysicien canadien, explique ce que signifie cette expression :

"Premier résultat, je dirais le plus dramatique : ça coupe le ciel, les gens ne voient plus le ciel. Vous avez des quantités de gens qui n'ont jamais vu la Voie lactée, qui n'ont jamais vu la lumière zodiacale. Des fois, je demande aux gens : est-ce que vous savez ce que c'est que la lumière zodiacale? Les trois quarts ne savent même pas, n'ont jamais entendu le mot. C'est quelque chose qui était très présent dans le passé. C'est ce contact avec le ciel, cette espèce d'émotion que vous avez quand vous sortez par une belle nuit étoilée avec la Voie lactée et tout. Ce contact, c'est quelque chose qui était présent dans toute I'humanité, jusqu'à peut-être quelques décennies, qui est absent et qui est quelque chose qu'il faut redonner aux gens $"^{3}$.

Ces halos de lumières artificielles ne nous permettent plus de contempler une partie de la nature. Ils nous éloignent de la réalité de notre monde, nous font oublier notre place au sein de notre Univers, que nous sommes des habitants de la planète Terre. Contempler le ciel nocturne est aussi plonger notre regard dans son infinité, souvent source de multiples questionnements.

De plus, la chasse continue faite à l'obscurité, cette obsession de l'omni-clarté et de la transparence ne nous permettrait plus de trouver le calme, le repos, le silence de la nuit [Sansot, 1997 ; Gaudin, 1991].

Aujourd'hui, il existerait un engouement de la population pour contempler à nouveau le ciel nocturne. La couleur brun-rouge régnant actuellement au-dessus des villes françaises durant la nuit et la rareté de la voûte noire étoilée constitue même la raison d'être de certains évènements comme la Nuit des Etoiles ou le Jour de la Nuit, soutenus par les astronomes. Le premier est organisé depuis 1991, par l'association Française d'Astronomie et Planète Science. Chaque été, des astronomes amateurs donnent rendez-vous gratuitement sur environ 300 lieux, pour observer la voie lactée. En 2008, cet évènement a réuni près de 100000 participants. Une Nuit des étoiles junior a ensuite été créée afin de faire découvrir le ciel aux enfants durant les heures sombres. Le second, impulsé par les associations Agir pour l'Environnement et l'ANPCEN, a eu lieu pour la première fois en octobre 2009, à l'occasion du passage à l'heure d'hiver. L'évènement a vocation "à sensibiliser les citoyens sur les nuisances engendrées par la pollution lumineuse et à

\footnotetext{
${ }^{3}$ http://astro-canada.ca/ fr/a3800.html
} 
renouer un lien avec la nuit noire et magique ${ }^{4}$. Plus de 160 villes ont participé dans l'ensemble du pays, dans lesquelles ont eu lieu des extinctions d'éclairage public, des animations astronomiques, des ballades nocturnes et, parfois, des animations en intérieur (conférence ou projections de films sur le ciel nocturne).

La pollution lumineuse modifie, incontestablement, les rapports de l'Homme à la Terre. De tels évènements rappellent l'artificialisation grandissante du monde qui l'éloigne de la nature. Par conséquent, cette pollution lumineuse qualifiée « d'astronomique " par Travis Longcore et Catherine Rich, n'est-elle pas, elle aussi, écologique ? Car l'écologie ne doitelle pas dorénavant questionner les rapports de l'Homme au monde, les liens que les sociétés entretiennent avec la nature, comme le pensent le physicien Jean-Paul Deléage [Deléage, 1991] ou le philosophe Thierry Paquot [Paquot, 2007] ?

L'expression "pollution lumineuse " recouvre ainsi diverses perturbations écologiques. Des questions se posent sur la disparition du ciel étoilé dans les villes et de l'obscurité, du noir propre à la nuit, qui a des répercutions sur les écosystèmes mais aussi des conséquences médicales, culturelles et anthropologiques.

Néanmoins, l'éclairage urbain constitue un véritable acquis et semble ne pouvoir être remis en cause sur certains points (sécurité routière, sentiment d'insécurité, qualité des espaces publics nocturnes, etc.). Il en résulte que la mise en éclairage intensive des villes suscite de nombreux débats. De quelles manières les collectivités territoriales comprennent-elles alors

la pollution lumineuse et tentent-elles de la réduire?

\section{Des solutions technicistes dans les collectivités territoriales}

La pollution lumineuse est considérée par les acteurs de l'éclairage au sein des collectivités selon deux axes principaux.

Le premier est relatif aux dépenses d'énergie. Le Plan Climat (plan d'actions mis en place par le ministère de l'écologie et du développement durable en 2004 afin de réduire l'émission des gaz à effet de serre) incite les collectivités à instaurer des plans climats territoriaux qui doivent s'inscrire au sein de leur Agenda 21 et à assurer une définition et une mise en œuvre d'objectifs. Paris a ainsi adopté son plan climat en octobre 2007 ceux de Plaine Commune et de la Gironde sont en cours d'élaboration. L'éclairage public se trouve au cœur de ces enjeux climatiques, en raison des importantes consommations énergétiques qu'il engendre : pour l'ensemble des communes françaises, il représente plus de $20 \%$ des dépenses énergétiques des communes et un peu moins de $50 \%$ de la consommation d'électricité d'une commune [Ademe, 2005]. Le plan climat de Paris prévoit alors une baisse de $30 \%$ de réduction des consommations énergétiques du parc municipal et de l'éclairage public entre 2004 et 2050.

Cependant, les personnes travaillant dans les collectivités territoriales disent elles-mêmes qu'elles en sont encore au "stade de départ " dans ce domaine, comme le signale un ingénieur-éclairagiste de la communauté d'agglomération Plaine Commune ${ }^{5}$ :

«Pour des raisons d'économies d'énergies, de préservation de l'environnement, on commence à réfléchir et se dire, bon, si on baisse, ça serait pas mal... $\|$ y a des technologies qui permettent de baisser l'intensité lumineuse : si on baisse de $30 \%$,

\footnotetext{
${ }^{4}$ Communiqué de presse du 22 octobre 2009

${ }^{5}$ Les compétences en matière de voierie et d'éclairage à Saint-Denis sont rassemblées au sein de cette communauté d'agglomération.
} 
$50 \%$, ça ne se verra pas forcément sur certains lieux. Et y'en a pas mal! (Rires) Mais on éclaire encore beaucoup $"^{6}$.

Réduire la " pollution lumineuse » est en fait d'abord vu comme une source d'économie financière. Les financements provenant des $\mathrm{EPCl}^{7}$ influencent beaucoup les décisions des communes ou des communautés de communes. Là encore, ce que nous dit cet éclairagiste est révélateur :

«Disons que le respect environnemental est pris en compte, parce qu'il permet de faire des économies financières, je vais vous le dire comme ça. Notamment avec le Sipperec : il a un volet éclairage public et nous impose certains critères matériels pour subventionner. II peut financer jusqu'à 50\%, donc ça nous influence beaucoup ".

En effet, le Sipperec (Syndicat Intercommunal de la Périphérie de Paris pour l'électricité et les Réseaux de Communication), qui regroupe plus de 80 villes situées dans les départements autour de Paris, possède des compétences en matière d'électricité et de télécommunication. Il accompagne des opérations de conseils et de maîtrise de l'énergie et subventionne des investissements dans le domaine de l'éclairage public. II peut également minorer les participations financières des communes faisant des "choix technologiques entraînant des économies dans les domaines de l'énergie et de l'exploitation » [http://www.sipperec.fr].

Généralement, cela se traduit, du côté des collectivités territoriales, par l'instauration de lampes de haute performance, de génération relativement récente, utilisant moins d'énergie, avec un meilleur rendement. A Paris, un plan de suppression des lampes à incandescence - très consommatrices d'énergie- a été établi entre 2003 et 2008 au profit de lampes à iodure métallique, qui ont une meilleure efficacité lumineuse et une durée de vie plus longue. Les lampes à vapeur de mercure, encore utilisées pour l'éclairage piétonnier, sont, elles aussi, en cours d'élimination, en application des directives européennes $^{8}$. Les actions se font aussi dans le domaine évènementiel. La ville opte de plus en plus pour les DEL (Diodes Electroluminescentes) durant les évènements majeurs comme les fêtes de fin d'année ou Paris Plages. Ces diodes permettent de réduire jusqu'à 70\% la consommation d'électricité. Le remplacement des traditionnelles illuminations des Champs-Elysées, durant les fêtes de fin d'année depuis 2007, au profit de lumières plus modernes et plus économiques en matière d'énergie a alors fait l'objet d'une surmédiatisation. Toutefois, certains lieux, comme les places de l'Hôtel de Ville et de l'Opéra sont encore éclairés par des lampes à incandescence à la demande des Architectes des Bâtiments de France, parce qu'elles offrent un excellent rendu des couleurs.

Cette politique générale ne signifie pas pour autant une baisse de l'intensité lumineuse : il est tout à fait possible d'utiliser des lampes de puissance moindre, tout en gardant un même éclairement au sol, voire en l'augmentant. A Paris, on garde ainsi volontairement la même quantité de lumière en éclairage. Limiter les dépenses énergétiques liées aux éclairages publics n'entretient donc aucun lien avec notre définition précédente de la pollution lumineuse...

\footnotetext{
${ }^{6}$ Entretien du 28 février 2008

${ }^{7}$ Etablissements Publics de Coopération Intercommunale

8 Voir la directive RoHS (Restriction of the use of certain Hasardous Substances in electrical and electronic equipment) de 2002 qui a pour objectif de réduire l'utilisation de substances dangereuses dans les équipements électriques et électroniques, ainsi que de la directive cadre EuP (Eco-design requirements for Energy Using Products) de 2005 qui vise à réduire les produits trop consommateurs d'énergie.
} 
Face à ce souci de réduction des dépenses financières et énergétiques, on peut tout de même noter certaines actions allant dans le sens d'une réduction des niveaux d'éclairement. Par exemple, une partie des autoroutes autour de Paris ne sera plus éclairée d'ici à 2010, suite à une décision de la Direction Interdépartementale des Routes d'lle-de-France (la Dirif). Cela est lié à un fait anecdotique : des voleurs de cuivre avaient dérobé une grande partie des câbles électriques de I'A15, qui traverse le Val-d'Oise, en 2007. La Dirif n'a pas remplacé ces câbles pour des raisons budgétaires et, deux années plus tard, elle remarque une baisse du nombre d'accidents de $30 \%$. Elle souligne alors que sécurité routière et éclairage ne vont pas forcément de pair : les lampes donneraient un sentiment de sécurité aux automobilistes qui rouleraient alors plus vite. Cela augmenterait même le risque d'accidents. Une partie des éclairages de l'A86, de l'A10 et de I'A13 doit alors être éteinte dès 2009. Au final, cela devrait permettre une baisse des coûts d'électricité de 20 à $25 \%$.

Le deuxième axe selon lequel est généralement définie la pollution lumineuse par les acteurs de l'éclairage urbain concerne la limitation de l'émission de lumière dans le ciel. II s'agit d'abord de bien diriger les éclairages. Là encore, les solutions sont ciblées sur le choix des luminaires. La protection du ciel nocturne intéressant directement les astronomes, I'ANPCEN fournit régulièrement des informations sur le sujet, comme le montre l'illustration ci-dessous.

\section{Informations de l'ANPCEN}

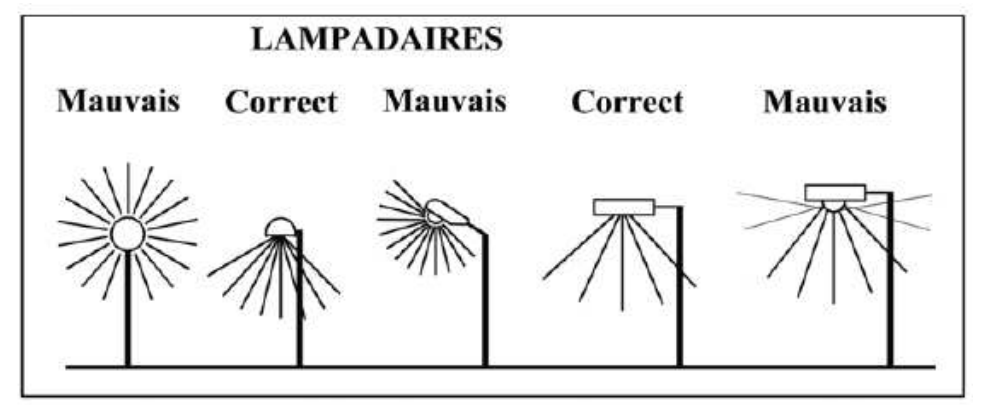

ANPCEN

Ainsi, les communes évitent de plus en plus d'utiliser des lampadaires de type " boule » et s'équipent progressivement de réflecteurs permettant d'orienter la lumière vers le bas. Néanmoins, si les actions sur la lumière directe sont aujourd'hui relativement bien contrôlées par les éclairagistes, les réflexions sur les surfaces -peu faciles à prévoir et difficiles à limiter- sont loin d'être maîtrisées.

\section{Une pollution souvent niée par les éclairagistes}

De nombreux acteurs de l'éclairage urbain considèrent, eux, que l'éclairage artificiel ne peut générer de pollution. Cela est souvent le positionnement pris par les éclairagistes, comme l'attestent ces propos d'un ingénieur de la Ville de Paris lors d'un entretien ${ }^{9}$ :

\footnotetext{
${ }^{9}$ Entretien du 10 mars 2006
} 
"L'éclairage ne se voit que parce qu'il y a des particules en suspension dans l'air. Hé oui ! La lumière rend juste visible la pollution de l'air ! C'est seulement parce qu'il y a déjà cette pollution qu'on peut voir un halo lumineux dans le ciel " ...

L'AFE $^{10}$ (Association Française de l'Eclairage), quant à elle, parle plutôt de « nuisances dues à la lumière " auxquelles elle a consacré un guide [AFE, 2006]. En préface de cet ouvrage, Christian Remande, ancien PDG de la société Comatelec et expert AFE nie toute existence d'une pollution lumineuse : "La lumière ne pollue pas, la lumière est invisible mais la lumière peut générer des nuisances qu'il y a lieu de ne pas occulter aujourd'hui. [...] l'éclairage peut générer un certain nombre de nuisances spécifiques qui ne concernent chacune qu'une catégorie particulière d'individus, d'animaux, de végétaux. C'est bien là que réside la très grande différence entre les pollutions de l'air et de l'eau (pour lesquelles les nuisances sont unanimement reconnues et combattues par tous) et la 'mal nommée pollution lumineuse' qui peut prendre des formes totalement différentes et ne toucher chaque fois qu'une fraction minoritaire de la population. Il est par conséquent, indispensable de distinguer et de classer les différentes formes de nuisances [...] plutôt que rassembler sous le terme générique de 'Pollution lumineuse' I'inventaire des constats négatifs relevés sur une minorité d'installations d'éclairage extérieur ». On peut aussi noter que le projet loi dit Grenelle 2, adopté le 8 octobre 2009, ne parle que de " nuisances lumineuses".

Pourtant, ces dites " nuisances " atteignent l'ensemble des êtres vivants et perturbent les écosystèmes. Et c'est justement pour ces raisons qu'il s'agit bien de pollution, puisque « le terme de nuisance désigne toute dégradation de l'environnement qui ne présente pas d'impact écotoxicologique mais qui a pour conséquence d'induire une gêne pour les personnes qui la subissent. A la différence des pollutions, les nuisances ne provoquent pas nécessairement d'effet néfaste sur la santé humaine et/ou sur le plan écologique. Toutefois, elles sont perçues à juste titre par ceux qui y sont exposés comme une modification défavorable de l'environnement » [Ramade, 2009].

De nombreuses confusions recouvrent ainsi l'expression "pollution lumineuse ", souvent niée par les éclairagistes ou prise comme synonyme de gaspillage énergétique. II en résulte que les solutions mises en avant par les collectivités sont parfois en décalage avec une définition de cette expression avant tout axée sur l'émission de lumière dans l'atmosphère. La protection actuelle du ciel nocturne réside principalement dans un traitement technique de l'éclairage, en tentant de maîtriser l'orientation des flux lumineux et de réduire, parfois, la quantité de lumière répandue dans le ciel.

Or, nous pensons que certaines questions demeurent pas ou peu posées à l'heure actuelle, pouvant certainement avoir un lien avec une meilleure maîtrise de la pollution lumineuse, notamment celle des usages de la ville nocturne.

\section{Environnement urbain nocturne : les enjeux peu explorés de l’urbanisme-lumière}

La lumière a directement participé à la création d'un nouveau temps urbain : la nuit urbaine [Paquot, 2000]. La création de l'éclairage public a provoqué de nombreux changements dans les modes de vie en ville. L'illumination fixe des rues, à l'huile puis au

\footnotetext{
${ }^{10}$ Regroupant principalement des ingénieurs et techniciens éclairagistes travaillant des entreprises privées ainsi que la plupart des éclairagistes des collectivités territoriales.
} 
gaz, a favorisé le développement de la vie urbaine nocturne. Dès le XVIIle siècle, "la nuit, jusque-là terra incognita, fut découverte et exploitée " [Schivelbusch, 1993] et l'habitude des fêtes tardives s'est développée. La lumière dessinait alors des géographies inédites. Elle touchait fortement à la façon dont les individus investissaient et pratiquaient l'espace urbain durant quelques heures.

Aujourd'hui encore, la nuit urbaine paraît ne pouvoir exister sans cet équipement et semble être source de présence humaine. Cependant, une analyse des politiques d'éclairage à l'échelle intra-urbaine montre combien celles-ci sont dorénavant déconnectées des usages de la ville.

\section{Des logiques fonctionnalistes persistantes}

Les collectivités territoriales font peu référence aux recherches existantes sur les usages et représentations nocturnes ${ }^{11}$ et peu d'études sont portées localement. Ce manque d'intérêt pour les vécus que la nuit urbaine suscite est particulièrement frappant à SaintDenis et plonge la ville dans une sorte de "cercle vicieux " en matière d'éclairage. La notion de sécurité guide ici essentiellement les aménagements. En effet, selon un chargé de mission "Prévention et tranquillité publique » de la Ville :

"Saint-Denis rencontre un taux de plaintes et de difficultés, notamment en matière de violences de voie publique, qui en font probablement la ville la plus violente de

France. [...] En tous cas, c'est une ville hors-norme sur une violence quotidienne $»^{12}$. Le taux de criminalité y est de l'ordre de plus de 160 pour mille en 2007, alors qu'en France métropolitaine il est estimé en moyenne à 58,33 pour 1000 habitants. II n'existe pas de données sur les relations entre la nuit et l'insécurité. Néanmoins, toutes les personnes travaillant sur la commune qui ont été interrogées rapportent qu'à la nuit tombée, un sentiment de malaise se fait ressentir, comme s'il existait un couvre-feu et qu'il règne un fort sentiment d'insécurité.

Face à ces constats généraux, les éclairagistes de Plaine Commune pensent d'abord qu'il faut fortement éclairer la ville. L'un deux explique ainsi, lors d'un entretien ${ }^{13}$ :

«On éclaire, on éclaire beaucoup. Sur un territoire comme le notre, il est inconcevable de ne pas éclairer. Je sais que dans certaines villes, des idées de passage par des zones d'ombres ou de jeux comme ça, ça se fait pas mal... Je l'ai vu à Berlin notamment, où il $y$ avait un traitement particulier de la lumière, en passant d'une zone éclairée à une zone qui l'est beaucoup moins. Ici ce sont des choses qui passeraient encore mal, pour un sentiment se sécurité tout simplement : on éclaire tout, on éclaire les squares qui sont fermés la nuit pour des raisons de sécurité, on éclaire un certain nombre de lieux... enfin on éclaire tout! On éclaire tout et beaucoup ".

Le nombre de sources lumineuses, tout d'abord, ne cesse de se multiplier : Saint-Denis est passée de 6400 points lumineux en 1989 à plus de 15000 en 2008. Ensuite, il semble s'imposer une augmentation incessante des niveaux d'éclairement, liée à la demande des résidents de la commune, ainsi que l'exprime un autre éclairagiste ${ }^{14}$ :

« Les mises en ambiance ? Bon, d'abord, il faut assurer la sécurité publique, donc déjà, il faut maintenir les niveaux d'éclairement, les gens sont de plus en plus exigeants, ils

\footnotetext{
${ }^{11}$ Comme, par exemple, celles menées par le CRESSON depuis le début des années 1990

${ }^{12}$ Entretien du 3 avril 2008

${ }^{13}$ Entretien du 28 février 2008

${ }^{14}$ Entretien du 18 mars 2008
} 
veulent de plus en plus... on est quasiment dans de la surenchère de lux. On éclaire par exemple les espaces piétons depuis bien longtemps à 20 lux. Or la réglementation ne nous impose pas de les éclairer à 20 lux mais à 7 lux, en gros... On impose cela aux aménageurs aussi. Or 20 lux, il s'avère que c'est insuffisant. [...] Cela passe par des pétitions, par ce qu'on entend dans des réunions de quartiers, donc on les inscrits dans le programme $"$.

Parfois, ce sont même les services de police qui jugent les éclairages insuffisants et influencent donc les décisions des élus et la mise en œuvre par les techniciens. Cela a été le cas pour le square Ile-de-France, zone de contact entre deux cités (la cité Floréal et la cité Soucet) et un quartier pavillonnaire. Les éclairages ont dus être changés en 2008 alors qu'ils avaient fait l'objet, environ cinq ans auparavant, d'une rénovation. Ce changement a été réalisé suite à une demande de la Direction de la Police Urbaine.

L'augmentation des niveaux d'éclairage semble alors être la principale, sinon l'unique solution offerte en ce qui concerne l'éclairage, aux problèmes liés à l'insécurité et au sentiment qui lui est associé.

Pourtant, les effets de l'augmentation du niveau lumineux sont loin d'être reconnus par les scientifiques. La plupart des recherches menées aux Etats-Unis, en Grande-Bretagne et en France sont peu fiables, parfois soutenues par le lobby des fabricants de luminaires et des fournisseurs d'énergie [Mosser, 2007]. Beaucoup de travaux se montrent peu objectifs et ont surtout servi à provoquer des débats. Toutefois certains points ont été repérés de façon assez consensuelle. De façon positive, l'éclairage accroît le risque pour un agresseur d'être vu ou pris sur le fait. De façon négative, il permet certaines activités, notamment pour les jeunes, qui peuvent gêner le voisinage durant son sommeil. II augmente aussi la visibilité des victimes et des objets leur appartenant ainsi que le nombre de personnes présentes dans les alentours pouvant s'interposer, ce qui peut par exemple favoriser les vols. Et la forte luminosité présente à un endroit restreint la visibilité des autres rues plus sombres, ce qui offre la possibilité aux agresseurs de s'enfuir de façon plus discrète.

En outre, une lumière forte peut engendrer des problèmes de dégradation. Par exemple, dans le quartier du Franc-Moisin, grand ensemble dont la construction a permis la résorption d'un important bidonville, situé à l'est du Stade de France, les luminaires se trouvent très fréquemment endommagés, car selon le chargé de mission "Prévention et tranquillité publique ", " elle dessert le business en cours " ${ }^{15}$ et c'est alors tout un quartier qui se trouve plongé dans l'obscurité.

Même l'éclairagiste travaillant depuis douze ans sur la ville de Saint-Denis se rend compte que "ce n'est pas en éclairant plus fort que l'on résout les problèmes et rassure les passants $\|^{16}$.

Il s'agit pourtant de l'unique réponse apportée face aux nombreuses plaintes des dionysiens qui pensent instinctivement que plus d'éclairage offre plus de sécurité. Cependant, aucun diagnostic, aucune expérimentation, aucune observation n'ont été réalisés sur la commune sur le rapport entre lumière et sécurité.

L'augmentation continue des niveaux d'intensité lumineuse met directement en question les coûts énergétiques dépensés et la pollution lumineuse générée. Toutefois, le sujet de la baisse de l'éclairage est dit "sensible " d'après un éclairagiste, et, même si l'on commence à réfléchir aux moyens de le réduire, cela se fait uniquement de façon

\footnotetext{
${ }^{15}$ Entretien du 3 avril 2008

${ }^{16}$ Entretien du 18 mars 2008
} 
ponctuelle dans certains endroits, peu ou pas fréquentés le soir comme les jardins publics et les zones d'activités.

Ainsi, Saint-Denis possède des éclairages très puissants mais qui ne semblent pas répondre à la demande sécuritaire faite par ses habitants. Les réflexes technicistes et fonctionnalistes qui ont dominé jusqu'au années 1980, loin d'avoir été éradiqués dominent encore nettement en réponse à la demande sécuritaire. Le cas de Saint-Denis témoigne d'un acharnement à vouloir traiter les espaces publics par un sur-clairement médiocre plutôt que de chercher des réponses dans le traitement des ambiances. II témoigne toutefois d'une logique encore dominante en France, allant à contre-courant de celle, écologique, consistant à limiter la pollution lumineuse.

\section{Un urbanisme-lumière peu préoccupé des usages populaires de l'éclairage nocturne}

Dans des villes toujours plus éclairées, les lieux les plus fréquentés ne sont pas, bien entendu, ceux les plus éclairés. Mais surtout, les lieux de vie nocturne ne sont pas ceux qui retiennent le plus l'attention des aménageurs en terme de lumière. II est très rare que ces endroits de la ville fassent l'objet d'une " mise en ambiance » nocturne.

A Bordeaux, le cas des quais de la rive gauche nous paraît être un exemple significatif. Les quais centraux bénéficient $d^{\prime}$ une ambitieuse politique de mise en lumière ${ }^{17}$ afin de mettre en valeur un panorama nocturne à contempler depuis l'autre rive. Quatre étages de lumière sont ainsi visibles: les berges, puis le boulevard et l'éclairage du tramway et, enfin, les façades des bâtiments. L'éclairage de la Place de la Bourse constitue le point d'orgue de cette politique. Réalisé par Alain Guilhot, l'éclairage se fait par 694 projecteurs sur 350 mètres de façade. Des projecteurs blancs en contre-plongée sont placés sous les trottoirs et intégrés aux façades, relevant les détails de l'architecture et la verticalité des pilastres. De petits projecteurs sont installés sur les jambages intérieurs des fenêtres. La silhouette de la toiture est soulignée par des réglettes fluorescentes et l'éclairage des clochetons.

A l'inverse, les quais de Paludate, situés au sud, en marge du centre-ville, font l'objet de peu d'attention. Ils regroupent pourtant une grande partie des discothèques, bars, restaurants de la ville ouverts jusque tard. Leurs nuits se sont animées depuis 1997, conséquence d'un important incendie ayant dévasté en grande partie les abattoirs, situés en face. Il existe alors un espace nocturne spécifique qui se dégage au sein de la ville, à l'écart du centre et relativement bien délimité, dans le secteur formé par les quais de Paludate, les alentours de la gare Saint-Jean et le pôle économique du marché de Brienne. Or ce secteur ne possède qu'un éclairage routier n'ayant bénéficié d'aucun traitement spécifique. Malgré les transformations du quartier, des activités qui ont fondamentalement changé, notamment sur les quais de Paludate, l'éclairage est resté le même. II n'est pas conçu pour le piéton et aucun travail sur les ambiances n'a été effectué. II n'existe pas, non plus, de projet en cours ou prévu allant dans ce sens. La fréquentation de l'espace nocturne y est cependant bien développée. Les quais de Palusdate et ses petites rues perpendiculaires réunissent souvent de nombreuses personnes qui discutent à la sortie ou l'entrée des boîtes de nuit donnant sur la rue.

\footnotetext{
${ }^{17}$ MAIRIE DE BORDEAUX, CONCEPTO (1996), Ville de Bordeaux. SDAL.
} 
L'éclairage privé vient alors pallier les carences de l'éclairage public. Ce sont les néons et enseignes lumineuses des restaurants, sandwicheries, bars, discothèques, cherchant avant tout à créer des lumières atypiques et originales pour leur devanture afin d'attirer l'œil du client, qui créent les ambiances lumineuses. Les néons multicolores contrastent radicalement avec les couleurs habituelles de la ville et de sa mise en lumière traditionnelle. Ces lumières, forcément tape-à-l'œil, cherchent à faire dans la démesure. Elles correspondent probablement à la nuit festive et exubérante où l'on se défoule, où l'on s'exhibe, où on " se lâche "...

Néanmoins, un éclairage de ce type suscite plusieurs questions. D'abord, on peut se demander s'il revient au privé, avec ses intérêts particuliers, de venir pallier un manque de l'éclairage public. Ensuite, on peut s'interroger sur la cohérence existante entre les éclairages publics et privés: ce secteur est doté d'un éclairage routier traditionnel puissant, ce qui provoque des situations de sur-éclairage à certains endroits. S'ajoute à cela que, bien entendu, ces commerces ne cherchent pas à limiter l'émission de leurs lumières vers le ciel. A ce jour, aucune législation ne vient réglementer l'émission de lumière provenant d'activités privées, qu'il s'agisse des enseignes ou de l'éclairage provenant des activités elles-mêmes (comme celles émanant des tours de bureaux...). Pourtant, l'éclairage public ne constitue qu'une partie des lumières nocturnes de la ville. N'y pas t'il pas de solutions à inventer de la part des communes ? Ne pourraient-elles pas imaginer, dans des quartiers de ce type, d'organiser une concertation avec les commerçants? Ne pourraient-elles pas inciter ceux-ci à réduire et mieux orienter leurs émissions de lumière tout en favorisant des éclairages festifs et attractifs dans le quartier ? A notre connaissance, aucune tentative de ce type n'a été réalisée à ce jour.

Les acteurs les plus actifs pour lutter contre la pollution lumineuse émise par les commerces et entreprises privées sont à trouver dans le domaine associatif. Certaines associations, notamment le Clan du Néon, militent pour l'extinction de certains éclairages. Cette dernière, créée en 2007, agit dans une dizaine de villes françaises. Ses actions se centrent sur l'extinction des néons allumés des boutiques fermées durant la nuit, dénoncés comme une "absurdité " [http://clanduneon.over-blog.com/]. Cela se fait de façon simple : il suffit de désactiver les boîtiers des néons, en principe placés à l'extérieur des magasins, en hauteur. Les membres de l'association notent sur leur site Internet : " [Nous] n'agissons jamais sur un commerce ouvert, un café ouvert, une pharmacie ouverte... [...] Le plus marquant étant le cas de certaines banques, dont les horaires sont restreints mais qui continuent d'afficher fièrement leur logo... de banque... la nuit. Des repères ? Oui mais trop de néons tuent le néon utile ! Trop de lumière éblouit ! Une enseigne indiquant un distributeur de billets, d'accord ; toute la banque allumée pour un distributeur de billets, non ". Deux raisons majeures sont ainsi évoquées : la soumission continuelle à une publicité non choisie ainsi que la génération de la pollution lumineuse.

\section{Quid de la concertation, de la consultation et de la participation?}

Aucune des trois collectivités étudiées n'a engagé de démarche de participation, de concertation ou de consultation auprès des habitants. Ce constat rejoint les analyses de Sophie Mosser montrant qu'au sein de l'urbanisme-lumière s'est formée une sorte de " doctrine opérationnelle ": depuis une vingtaine d'années, un discours s'est construit sur ce que doit être et faire l'éclairage public, alors qu'il existe peu d'informations sur la façon dont il est vécu au quotidien [Mosser, 2005]. Ce discours repose essentiellement sur 
l'expérience et la créativité des praticiens et concepteurs-lumière. L'engouement général pour l'urbanisme-lumière a fait naître une sorte d'assurance sur ce que doit être la production de la ville nocturne, qui repose sur peu d'incertitudes et de remises en questions. Au final, malgré de bonnes intentions, "l'analyse des processus d'élaboration de politiques et de projets montre plutôt des effets complexes de composition, voire des effets pervers, au sens de R. Boudon. [...]. Les incertitudes sont d'autant plus fortes, pour l'éclairage, qu'il s'agit d'un domaine pour lequel les dispositifs de maîtrise programmatique sont faibles (programmes concis, cahiers des charges succincts, dispositifs de coordination des acteurs rares, traitement de l'éclairage repoussé en fin de projet de chantier) " [Mosser, 2005].

L'évaluation et la satisfaction de l'éclairage par les citadins est complexe et dépend d'une multiplicité de critères liés à de nombreux éléments du quartier, relevant de l'environnement physique et social [Glodt, 2006]. Or on peut constater, vingt ans après l'apparition de l'urbanisme-lumière, que peu de dispositifs de concertation ou d'évaluation ont été conduits bien qu'ils commencent maintenant à se diffuser, comme le témoigne en particulier l'ouvrage dirigé par Jean-Michel Deleuil Eclairer la ville autrement. Innovations et expérimentations en éclairage public publié en 2009. Les enquêtes menées auprès des usagers du mail d'Empalot à Toulouse par des sociologues associés à un concepteur-lumière montrent ainsi que l'adaptation des éclairages aux cheminements des piétons et à leur perception de l'espace ne vont pas forcément " de soi " et que la traduction des demandes ou propositions des enquêtés en réalisation technique n'est guère aisée et nécessite un véritable travail d'interprétation [Fiori, Leroux, Narboni, 2009]. De façon générale, on peut supposer qu'une meilleure compréhension des attentes des usagers pourrait permettre de réduire les éclairages dans certains lieux.

\section{Un urbanisme-lumière peu soucieux des usages et vécus des individus}

Depuis la fin des années 1980, les discours liés à l'urbanisme-lumière affichent une volonté de valoriser les spécificités de la nuit, une importance grandissante accordée aux ambiances nocturnes, une préoccupation nouvelle au vécu des usagers. Cependant, malgré quelques expériences récentes visant à tenir compte des pratiques des espaces publics, les apports de l'urbanisme-lumière restent principalement liés aux mises en lumière patrimoniales [Mallet, 2009]. A Bordeaux, par exemple, le SDAL s'attache surtout à faire ressortir les lieux monumentaux de la ville et concerne avant tout la partie historique. La nuit est d'abord vue comme une "masse noire ", une obscurité malléable permettant de sélectionner les éléments sensés être regardés. L'obscurité de la nuit, permettant d'exploiter les propriétés de la lumière, est devenue un véritable atout en matière de communication pour les villes. Cette obscurité permet aux pouvoirs publics et aménageurs, qui ont réalisé de nombreux progrès dans la maîtrise technique de la lumière, de transformer la vision traditionnelle de la ville et de modeler une sorte de paysage idéal. Au final, l'attention est surtout portée au noir, à l'ombre, et non à la nuit urbaine, en tant que réalité temporelle particulière, suscitant des vécus, usages et perceptions spécifiques. C'est donc d'abord un paysage que l'on cherche à aménager compris dans un sens restrictif : un cadre fait pour être regardé, sorte d'image à laquelle le passant reste extérieur. 
Les pratiques renouvelées en matière d'éclairage urbain, qui entendent " magnifier la nuit ${ }^{18}$ et réaliser des "schémas d'aménagement nocturnes ${ }^{19}$ s'attachent peu aux différents usages des espaces publics nocturnes et au vécu des individus. De nombreuses questions sur l'environnement urbain nocturne, intégrant l'ensemble des aspects physiques, sensibles, pratiques, sociaux d'une ville dynamique, sans cesse en recomposition, restent encore peu posées.

Aux définitions floues de la pollution lumineuse répondent ainsi des solutions réduites à des préconisations quasi-exclusivement techniques et semblables d'une ville à l'autre. Pourtant, la réduction de cette pollution ne pourrait-elle pas passer par une analyse fine des usages et vécus de la ville nocturne ? Ne faut-il pas passer de la prise en compte du paysage-lumière à celle de l'environnement nocturne dans son ensemble ? Ou doit-on compter sur une future généralisation des éclairages à détecteur de présence dans l'ensemble des villes françaises afin de pouvoir concilier un peu mieux usages et éclairages?

\section{Bibliographie}

ADEME. 2005. Energie et patrimoine communal, enquête 2005, synthèse, 26 p.

AFE. 2006. Les nuisances dues à la lumière, Paris, Lux, 95 p.

AUGOYARD, Jean-François (dir.) (1990), Les facteurs lumineux du sentiment d'insécurité, séminaire de recherche exploratoire, $112 \mathrm{p}$.

BELLAVOINE, Christine. 2005. Les soirées à Saint-Denis, enquête auprès de dionysiens

CINZANO, P. (sous la direction de). 2002. Light pollution and the protection of the night environment, Instituto di scienza e tecnologia dell'inquinamento luminoso, $356 \mathrm{p}$.

DELEAGE, J-P. 1991. Une histoire de l'écologie, rééd. 1994, Paris, Seuil, 330 p.

FIORI, S. ; REGNAULT, C. 2006. Concepteurs sonores et concepteurs lumière, rapport du laboratoire CRESSON, $247 \mathrm{p}$.

FIORI, S.; LEROUX, M.; NARBONI, R. 2009. "La conception d'ambiances nocturnes : de l'enquête sociologique au projet lumière ", In : DELEUIL, J.-M. (dir.). 2009. Eclairer la ville autrement: innovations et expérimentations en éclairage public, Lausanne, Presses polytechniques et universitaires romandes, $295 \mathrm{p}$.

GAUDIN, H. 1991. "La chasse aux ombres", Autrement série mutations, novembre 1991, n¹25, pp. 63-71.

GLODT, H. 2006. Qualité perçue de l'éclairage urbain, dans la ville, la nuit, thèse de doctorat en psychologie de l'environnement soutenue à Paris X Nanterre, sous la direction d'Annie Moch, 318 p.

GWIAZDZINSKI, L. 2002. La nuit dimension oubliée de la ville, entre insécurité et animation, Thèse de doctorat en géographie, Faculté de géographie de Strasbourg sous la direction de Colette Cauvin-Reymond, 761 p., deux volumes annexes.

KWIATKOWSKI F. (et Al.). 2004. "Stress, cancer et rythme circadien de la mélatonine", Pathologie Biologie, $\mathrm{n}^{\circ} 53$, pp. 269-272.

LONGCORE, T. ; RICH, C. 2004. "Ecological light pollution", Frontiers in Ecology and the Environment, n², pp.191-198.

\footnotetext{
${ }^{18}$ Discours d'Alain Juppé [Lux, 2001]

${ }^{19}$ Mairie de Paris (2000), Schéma lumière d'aménagement nocturne du site de la Seine, décembre 2000, 96 p.
} 
"Bordeaux. Le plan lumière cinq ans après... ", Lux, août 2001.

MALLET, S. 2009. De l'urbanisme-lumière à la chronotopie. Vers un urbanisme temporel. Thèse de doctorat en urbanisme, Institut d'Urbanisme de Paris, Université Paris Est-Paris 12 Val-de-Marne, sous la direction de Thierry Paquot, $474 \mathrm{p}$.

MALLET, S. 2010. "Exposer les espaces référents d'une politique urbaine : le cas des mises en lumière à Bordeaux ", Lieux communs, $n^{\circ} 13$, juin 2010.

MOSSER, S. 2005. "Les configurations lumineuses de la ville la nuit: quelle construction sociale ? ", Espace et sociétés, $3^{\circ}$ trimestre, $\mathrm{n}^{\circ} 122$.

MOSSER, S. 2007. "Eclairage et sécurité en ville: I'état des savoirs ", Déviance et société, $\mathrm{n}^{\circ} 31$, janvier 2007, pp. 77-100.

NARBONI, R. 1997. La lumière urbaine, éclairer les espaces publics, Paris, Le Moniteur, 264 p.

NARBONI, R. 2003. La lumière et le paysage, créer des paysages nocturnes, Paris, Le Moniteur, $230 \mathrm{p}$.

NARBONI, R. 1998. Le paysage lumière : pour une politique qualitative de l'éclairage urbain, Paris, CERTU, $156 \mathrm{p}$.

PAQUOT, Th. 2000. "Le sentiment de la nuit urbaine aux XIXe et XXe siècles ", Les Annales de la recherche urbaine, septembre 2000, $\mathrm{n}^{\circ} 87, \mathrm{pp} .6-14$.

PAQUOT, Th. 2007. Petit manifeste pour une écologie existentielle, Paris, Bourin Editeur, $119 \mathrm{p}$.

RAMADE, F. 2009. "Pollution », Encyclopaedia Universalis.

SANSOT, P. 1997. "Un temps contrasté », Information sociales, $\mathrm{n}^{\circ} 29, \mathrm{pp} .10-15$.

SCHIVELBUSCH, W. 1983, traduit de l'allemand, 1993. La nuit désenchantée, Paris, Le promeneur, $199 \mathrm{p}$. 